\title{
6U CubeSat design for Earth observation with 6.5m GSD, five spectral bands and 14Mbps downlink
}

\author{
S. R. Tsitas \\ s.r.tsitas@alumni2008.cranfield.ac.uk \\ J. Kingston \\ j.kingston@cranfield.ac.uk \\ Cranfield Space Research Centre, Aerospace Engineering Department \\ School of Engineering, Cranfield University \\ Cranfield, UK
}

\begin{abstract}
The design of a $6 \mathrm{U}$ CubeSat including spacecraft systems and imaging payload is described for an Earth observation mission. From a Sun synchronous orbit at an altitude of $600 \mathrm{~km}$ the design enables imaging with a $6.5 \mathrm{~m} \mathrm{GSD}$, an optical MTF (on axis) of $>59 \%$ at half Nyquist and $>35 \%$ at Nyquist, a $26 \mathrm{~km}$ swath, 12 bit digitisation and SNR of 120-200:one in five spectral bands; blue, green, red, red edge and near infrared. Data can be downlinked at the rate of $14 \mathrm{Mbps}$ to a $3.7 \mathrm{~m} \mathrm{~S}$ band ground station. This design allows an $8 \mathrm{~kg}$ CubeSat to perform Earth observation missions equivalent to those of current 50$150 \mathrm{~kg}$ microsatellites, with a corresponding reduction in cost.
\end{abstract}

\section{NOMENCLATURE}

$A \quad$ satellite cross-sectional area in the direction of travel

$B \quad$ ballistic coefficient

$c \quad$ speed of light

$C_{D} \quad$ satellite drag coefficient

$d \quad$ pixel width

$D_{o} \quad$ telescope obstruction diameter

$D \quad$ imager aperture diameter

$\mathrm{Eb} \quad$ received energy per bit

$f \quad$ telescope focal length

$G_{T} \quad$ satellite antenna gain

$G / T \quad$ figure of merit

$h \quad$ Planck's constant

$H \quad$ altitude

$k \quad$ Boltzmann's constant

$l \quad$ separation distance between two resolvable points

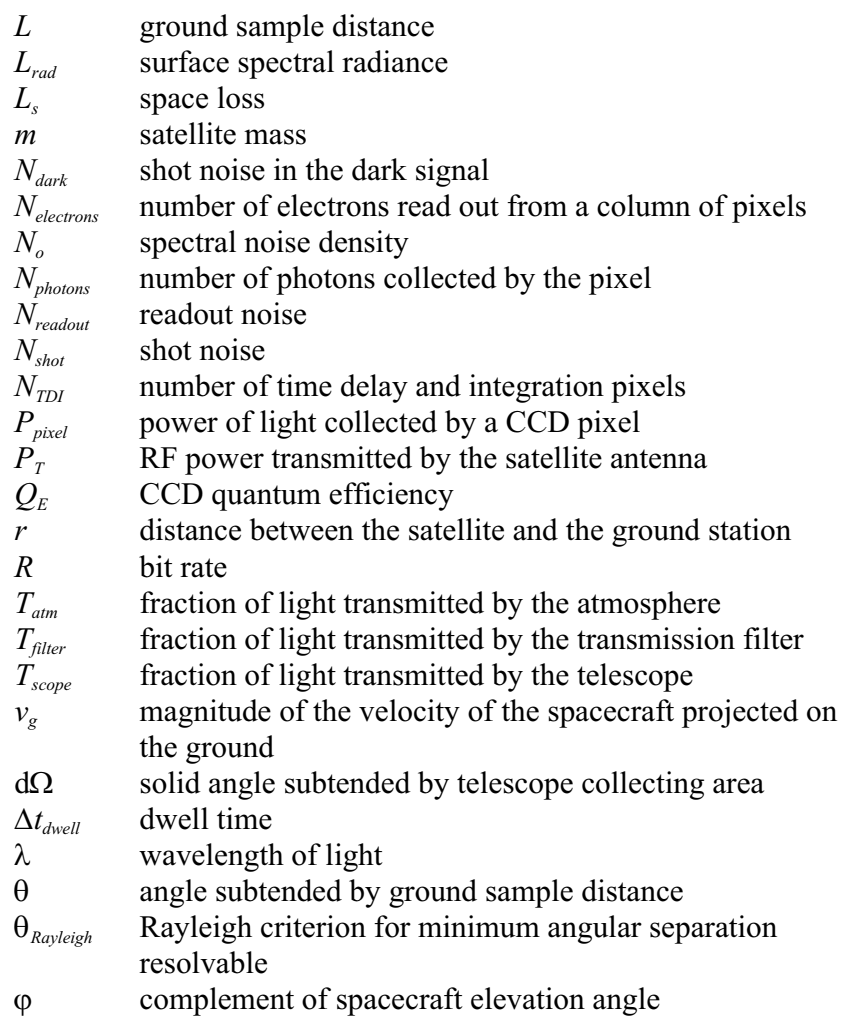


Table 1

Capabilities of the DMC, NigeriaSat-2 and RapidEye spacecraft

\begin{tabular}{|c|c|c|c|c|c|}
\hline Spacecraft & Mass & Nadir GSD/Swath/Spectral bands & Optical MTF & Digitisation & Downlink rat \\
\hline $\begin{array}{l}\text { DMC } \\
\text { (First generation) }\end{array}$ & $100 \mathrm{~kg}$ & $32 \mathrm{~m} / 600 \mathrm{~km} / 3$ bands & $\begin{array}{l}\text { (a) } 1 / 2 \text { Nyquist (on axis) }>60 \% \\
\text { @ Nyquist }>20 \%{ }^{(9)}\end{array}$ & 8 bits & $8 \mathrm{Mbps}$ \\
\hline $\begin{array}{l}\text { NigeriaSat }-2 \\
\text { (SSTL) }\end{array}$ & $300 \mathrm{~kg}$ & $\begin{array}{l}32 \mathrm{~m} / 300 \mathrm{~km} / 4 \text { bands } \\
2 \cdot 5 \mathrm{~m} / 20 \mathrm{~km} / \mathrm{PAN} \\
5 \mathrm{~m} / 20 \mathrm{~km} / 4 \text { bands }\end{array}$ & & $8-10$ bits & $2 \times 105 \mathrm{Mbps}$ \\
\hline
\end{tabular}

DMC $\quad 120 \mathrm{~kg} \quad 22 \mathrm{~m} / 600 \mathrm{~km} / 3$ bands

(Second generation)

$8-10$ bits

$40 \mathrm{Mbps}$

RapidEye $\quad 150 \mathrm{~kg} \quad 6.5 \mathrm{~m} / 77 \mathrm{~km} / 5$ bands

(a) $1 / 2$ Nyquist $=63 \%$

(a) Nyquist $=24 \cdot 5 \%{ }^{(10)}$

12 bits

$>60 \mathrm{Mbps}$

\subsection{INTRODUCTION}

Advances in CCD detectors and communication error correction codes combined with a compact long focal length telescope and the availability of compact star trackers, RWAs and fiber optic gyroscopes make it possible for an $8 \mathrm{~kg}$ nanosatellite to carry out the commercial Earth observation mission of a microsatellite. The system design of such a nanosatellite, based on a logical extension of the CubeSat standard to $6 \mathrm{U}(\sim 30 \mathrm{~cm} \times 20 \mathrm{~cm} \times 10 \mathrm{~cm})$, is described in this paper.

\subsection{LAUNCH AND ORBIT (600KM SUN SYNCHRONOUS ORBIT)}

CubeSats are deployed from P-POD launch tubes typically mounted on the payload interface of the launcher upper stage $e^{(1,2)}$. There are proposals for $6 \mathrm{U}$ P-POD dispensers ${ }^{(3)}$ to enable the launch of $\sim 30 \mathrm{~cm}$ $\times 20 \mathrm{~cm} \times 10 \mathrm{~cm}$ CubeSats which are big enough for the design described in this paper. One of the attractions of the CubeSat standard is the launch opportunities afforded by the widening use of P-PODs. It is reasonable to assume that if an energetic (i.e. nonelectric) propulsion system is included on a $6 \mathrm{U}$ CubeSat launch opportunities would be reduced. A propulsion system based on a gas stored under pressure, or an otherwise energetic system (for example bipropellant based) could jeopardise the launcher or the primary payload. The main launch customer or their insurer could take issue with the risk this creates.

Until electric propulsion systems are available for CubeSats, avoiding loss of launch opportunities creates the requirement that the $6 \mathrm{U}$ CubeSat have no propulsion system. Maintaining constant local time of the ascending node is a requirement for Earth observation. This ensures that all images are acquired under similar lighting conditions so that physical changes can be more easily discerned. To satisfy these orbit requirements without propulsion, the example of the SSTL Disaster Monitoring Constellation is followed. The DMC satellites do not use propulsion to maintain constant LTAN $^{(4)}$, relying instead on a suitable altitude $(\sim 700 \mathrm{~km})$ and inclination (Sun synchronous orbit) and this approach is used for the $6 \mathrm{U}$ CubeSat.

This approach has been validated by the experience of the PROBA-1 spacecraft which does not have a propulsion system. It was launched into a $681 \times 561 \mathrm{~km}$ Sun synchronous orbit with an initial LTAN of 10:30 AM. The LTAN drifted to 10:46 AM after three years in orbit, and the altitude decayed by less than $14 \mathrm{~km}^{(5)}$. This is close enough to constant LTAN to meet requirements during a typical mission lifetime.

The ballistic coefficient, $B$, of a satellite is given by Equation (1) where $m$ is the satellite mass, $A$ is the cross-sectional area in the direction of flight and $C_{D}$ is the drag coefficient.

$$
B=\frac{m}{A C_{D}}
$$

For the 6U CubeSat $m \sim 8 \mathrm{~kg}, A=0.0367 \mathrm{~m}^{2}$ (assumed to be tumbling at end of life, so the average area of the six faces is used), $C_{D} \sim 2$ and $B=109 \mathrm{kgm}^{-2}$. The lifetime of this satellite in a circular orbit at an altitude of $600 \mathrm{~km}$ can be estimated from Figs 3-17 of Wertz ${ }^{(6)}$ to be 12 years, however it is noted that this estimate can be in error by $50 \%$ due to changes in the atmospheric density due to solar activity. Along with the experience of PROBA-1, this indicates that altitude maintenance during a short mission is not a concern, and that the lifetime could possibly exceed the maximum of 25 years allowed to satisfy orbital debris mitigation requirements ${ }^{(7)}$. This leads to the requirement for a deorbit device. A deployable tether designed for CubeSats ${ }^{(8)}$ is specified for the deorbit device. With dimensions of $100 \mathrm{~mm} \times 83 \mathrm{~mm} \times 5 \mathrm{~mm}$ and a mass $<80$ grams the deorbit device fits on one $\sim 30 \mathrm{~cm} \times 10 \mathrm{~cm}$ face of the $6 \mathrm{U}$ CubeSat.

\subsection{PAYLOAD}

The requirement for the $6 \mathrm{U}$ CubeSat imaging payload is that it generates commercially useful data. The DMC and RapidEye Earth Observation spacecraft generate commercially useful data in spectral bands and at GSDs feasible for the 6U CubeSat to emulate so these are used as benchmarks. The requirement for the $6 \mathrm{U}$ CubeSat imaging payload may therefore be restated as that it is competitive with the DMC and RapidEye Earth Observation spacecraft. The capabilities of these spacecraft are summarised in Table 1. NigeriaSat-2, also built by SSTL, is included to demonstrate the capabilities of the SSTL high resolution VHRI imager which it carries.

The spectral bands used by RapidEye ${ }^{(11)}$ are taken as the requirements for the spectral bands imaged by the $6 \mathrm{U}$ CubeSat. SNR requirements are obtained from the GMES Sentinel-2 Mission Requirements ${ }^{(12)}$ in five spectral bands close to the bands imaged by RapidEye. To satisfy requirements the $6 \mathrm{U}$ CubeSat is specified to have the parameters given in Table 2 .

\subsection{Questar 3.5 telescope satisfies requirements for GSD and MTF}

It may be seen that a large aperture is not required to achieve adequate spatial resolution by considering the Rayleigh criterion for the resolution of two bright points which is given by Equation (2). $D$ is the aperture diameter and $\lambda$ is the wavelength of the light. 
Table 2

6U CubeSat parameters which meet requirements

\begin{tabular}{|c|c|c|c|c|c|c|c|c|}
\hline $\begin{array}{l}\text { Altitude } \\
\text { and orbit }\end{array}$ & $\begin{array}{l}\text { Nadir } \\
\text { GSD }\end{array}$ & Swath & $\begin{array}{l}\text { Spectral } \\
\text { bands }\end{array}$ & $\begin{array}{l}\text { Optical } \\
\text { MTF }\end{array}$ & $\begin{array}{l}\text { Imager } \\
\text { MTF }\end{array}$ & Digitisation & SNR & $\begin{array}{l}\text { Downlink } \\
\text { rate* }\end{array}$ \\
\hline $\begin{array}{l}600 \mathrm{~km} \\
\text { Sun } \\
\text { synchronous }\end{array}$ & $6.5 \mathrm{~m}$ & $>25 \mathrm{~km}$ & $\begin{array}{l}440-510 n \\
520-590 n \\
630-685 n \\
690-730 n \\
760-850 n\end{array}$ & & & 12 bits & $\begin{array}{l}154 \\
168 \cdot 4 \\
142 \\
117 \\
174 \cdot 6\end{array}$ & $>10 \mathrm{Mbps}$ \\
\hline
\end{tabular}

* To a 3.7 metre ground station

(a) $1 / 2$ Nyquist $(0 \cdot 7$ field $)>55 \%$

(a) Nyquist ( 0.7 field $)>20 \%$

(a) Nyquist ( 0.7 field $) \geq 7 \%$

$\theta_{\text {Rayleigh }}=\frac{1 \cdot 22 \lambda}{D}$

If the points are separated by a distance $l$ and viewed from an altitude $H$ then the minimum separation distance for which the points can be resolved according to the Rayleigh criterion is given by Equation (3).

$D=\frac{1 \cdot 22 \lambda H}{l}$

For $\lambda=550 \mathrm{~nm}$ representing visible imaging and $H=600 \mathrm{~km}$, the expression for $D$ reduces to Equation (4):

$D=\frac{40}{l(m)} \mathrm{cm}$

Therefore an aperture of $10 \mathrm{~cm}$ is able to resolve two points $4.0 \mathrm{~m}$ apart from an altitude of $600 \mathrm{~km}$. This presents a prima facie case that a small aperture telescope can be used to provide commercial Earth observation competitive with the DMC-2 and RapidEye spacecraft.

Sufficient contrast at medium and high spatial frequencies is required for high quality images of non-point sources. This is quantified by the modulation transfer function (MTF). To satisfy the daughter requirements for GSD, swath width and MTF given in Table 2, a telescope with a good MTF across a reasonably wide field of view and long focal length in a compact package is required. The Questar 3.5 telescope has an aperture of $89 \mathrm{~mm}$ and an effective focal length of $1,400 \mathrm{~mm}^{(13)}$. The optical tube assembly is $20.3 \mathrm{~cm}$ long fwith a mass of $1.4 \mathrm{~kg}^{(14)}$. These dimensions allow the OTA to fit within the $3 \mathrm{U}(\sim 30 \mathrm{~cm} \times 10 \mathrm{~cm} \times 10 \mathrm{~cm})$ volume allocated to the payload in the 6U CubeSat (Fig. 2). Despite the compact size the telescope has a relatively long focal length due to the optical design.

A long focal length is important to ensure sufficiently large CCD pixels. If the pixels are too small a suitable CCD may not be available. The pixel size is given by Equation (5) where $f$ is the telescope focal length, $L$ is the ground sample distance (GSD), $\mathrm{H}$ is the altitude and $d$ is the pixel width.

$$
d=f \frac{L}{H}
$$

For $f=1,400 \mathrm{~mm}, L=6.5 \mathrm{~m}$ and $H=600 \mathrm{~km}, d=15 \mu \mathrm{m}$ which is comfortably larger than the smallest available CCD pixel widths indicating a shorter focal length may be used. Shorter focal lengths require smaller telescopes. A CCD with $8.75 \mu \mathrm{m}$ pixels will be used as discussed in the next section. For $d=8.75 \mu \mathrm{m}, L=6.5 \mathrm{~m}$ and $H=$ $600 \mathrm{~km}, f=808 \mathrm{~mm}$. The highest spatial frequency in the ground image that can be reliably reconstructed is the Nyquist frequency, at which the image of one cycle of an alternating pattern of light and dark lines on the ground falls exactly within two pixels in the focal

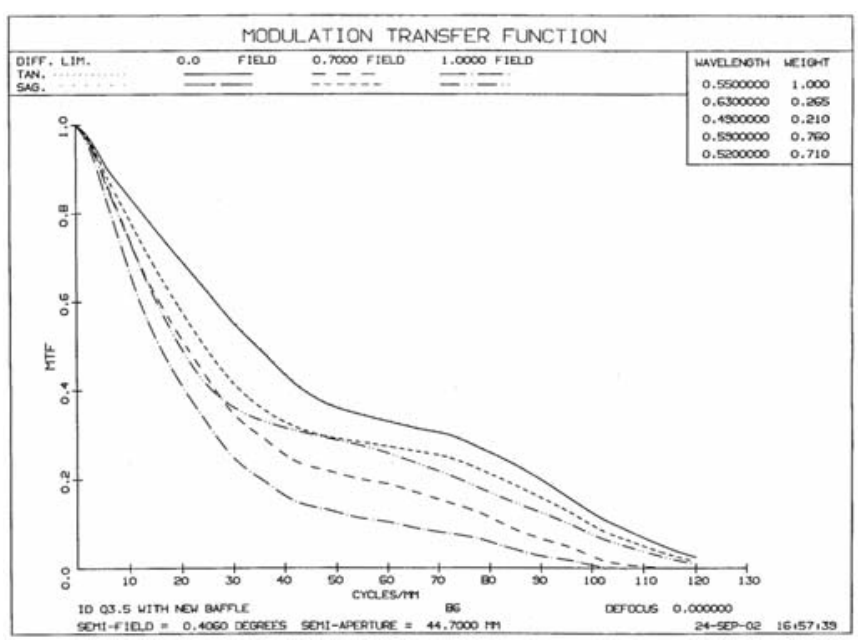

Figure 1. Modulation transfer function of the Questar 3.5 with wavelength weightings for visual observation and $18 \mathrm{~mm}$ image diameter ${ }^{(15)}$.

plane. As there are 114.3 pixels per millimetre for $8.75 \mu \mathrm{m}$ pixels, the Nyquist frequency is $57 \cdot 1$ line pairs per millimetre. Higher spatial frequencies in the ground image will lead to artifacts in the sampled imaged. The spatial resolution is therefore twice the GSD, or $13 \mathrm{~m}$, which is appreciably coarser than the resolution predicted by the Rayleigh criterion of $4.5 \mathrm{~m}$. This suggests sufficient values for the MTF might be achieved.

The modulation transfer function for the Questar $3.5(f=1,400 \mathrm{~mm}$, $\mathrm{f} / 16$ ) with wavelength weightings appropriate for visual observation and $18 \mathrm{~mm}$ image diameter $\left(0.74^{\circ}\right.$ field of view) has been provided by Questar Corporation ${ }^{(15)}$ and is shown in Fig. 1. The curve is not expected to be significantly different if weightings for the blue, green and red spectral bands matching the response of the CCD detector discussed in the next section are used. The MTF on axis and at 0.7 and 1.0 field ( $70 \%$ and $100 \%$ towards the edge of the field of view) in the sagittal direction are of interest. Since the CCD is essentially linear it will be aligned along the sagittal direction since the MTF is higher in the sagittal direction than the tangential direction. The MTF values on axis and at 0.7 and 1.0 field at half the Nyquist frequency and at the Nyquist frequency are summarised in Table 3.

A bespoke design staying within the dimensions of the off the shelf model and retaining the $1,400 \mathrm{~mm}$ focal length could match or improve these MTF values but with an image size of up to $36 \mathrm{~mm}^{(16)}$ $\left(1.5^{\circ}\right.$ field of view). For a constant aperture diameter the field of view of a telescope is approximately inversely proportional to the focal length. Therefore an $808 \mathrm{~mm}$ focal length version of the Questar 3.5 (f/9) will have at least a $2.5^{\circ}$ field of view, giving a swath width of $26 \mathrm{~km}$ at $600 \mathrm{~km}$ altitude which meets requirements. 
Table 3

Questar 3.5 MTF on axis and at 0.7 and 1.0 field from Fig. 1

$\begin{array}{lccc}\text { Spatial frequency } & \text { MTF on axis } & \text { MTF 0.7 field } & \text { MTF 1.0 field } \\ \begin{array}{l}1 / 2 \text { Nyquist } \\ (28.6 \text { cycles } / \mathrm{mm})\end{array} & 59 \% & 45 \% & 39 \% \\ \text { Nyquist } & 35 \% & 30 \% & 28 \%\end{array}$

$(57 \cdot 1$ cycles $/ \mathrm{mm})$

The MTF curve of an $808 \mathrm{~mm}$ focal length version of the Questar 3.5 will be different from the MTF curve for the $1,400 \mathrm{~mm}$ focal length model given in Fig. 1. However, the values given in Table 3 will be lower limits for the MTF values of an $808 \mathrm{~mm}$ focal length version at these spatial frequencies which meets requirements for optical MTF.

The imager MTF is the product of the optical MTF with the MTF due to detector geometry, read out sampling, image smear due to motion of the imager as light for a pixel is collected and optical tolerances respectively. Representative values of 0.64, 0.64, 0.8 and 0.7 respectively are used for these factors. Multiplying the MTF at Nyquist at 0.7 field with these values yields an imager MTF of $7 \%$, meeting requirements.

The Questar 3.5 telescope has spaceflight heritage dating back to 1966, when it was successfully used in two Aerobee 150 rocket flights $^{(17)}$. Verdone ${ }^{(17)}$ does not quantify the changes to mass and volume of the Questar 3.5 necessary to adapt the telescope to spaceflight. However from the description it is clear that the changes necessary to adapt the telescope to the mechanical, thermal and vacuum environment of launch and spaceflight did not add significantly to the mass or volume of the telescope.

\subsection{TDI CCD increases SNR to compensate for smaller aperture}

The use of a time delay and integration (TDI) CCD improves the SNR, allowing a smaller aperture to achieve the SNR of a larger aperture. Pushbroom imaging from a satellite relies on a linear CCD of usually a few thousand pixels, oriented across track. A TDI CCD extends the linear CCD in the direction along track with multiple rows, on the order of 100 or less. The electrons generated in pixels in the first row due to illumination by the image are transferred to the next row in time with the image shifting to the next row as the spacecraft moves forward. In this way the signal is built up by effectively increasing the exposure time.

The Fairchild Imaging CCD5061 is a 6,144 pixel TDI CCD with $8.75 \mu \mathrm{m}$ pixels and $4,8,16,32,64,96$ or 128 selectable TDI stages ${ }^{(18)}$. With a GSD of $6.5 \mathrm{~m}$ and swath width of $26 \mathrm{~km}, 4,000$ pixels will be illuminated by the ground image.

\subsection{SNR calculation: SNR requirements met for 5 required spectral bands}

The solid angle, $d \Omega$, subtended by the actual collecting area of a telescope of diameter $D$ with a central obstruction $D_{o}$ at an altitude $H$ is given by Equation (6):

$d \Omega=\frac{\pi\left(D^{2}-D_{o}^{2}\right)}{4 H^{2}}$

The power of light scattered from a square area of side length $L$ on the Earth's surface collected by a CCD pixel behind a transmission filter at the telescope's focal plane in the wavelength interval $\Delta \lambda$ about a wavelength $\lambda$ is given by Equation (7). $\varphi$ is the angle between the normal to the Earth's surface and the direction to the telescope, $T_{\text {scope }}$ is the transmission factor of the telescope, $T_{a t m}$ is the transmission factor of the atmosphere, $T_{\text {filter }}$ is the transmission factor of the transmission filter which will be used to separate the light incident upon the focal plane into different wavelengths and $L_{\text {rad }}$ is the surface spectral radiance.

$$
P_{\text {pixel }}=T_{\text {atm }} T_{\text {scope }} T_{\text {filter }} L_{\text {rad }} L^{2} \operatorname{Cos} \phi d \Omega \Delta \lambda
$$

The surface spectral radiance is the flux of light scattered from the Earth's surface per unit steradian per unit wavelength. In a pushbroom imager the light is collected by a pixel for the dwell time $\Delta t_{\text {dwell }}$ given by Equation (8) where $v_{g}$ is the magnitude of the velocity of the spacecraft projected on the ground.

$$
\Delta t_{d w e l l}=\frac{L}{v_{g}}
$$

The number of photons collected by the pixel scattered from the ground element of side length $L$ is given by Equation (9) where $h$ is Planck's constant and $c$ is the speed of light.

$N_{\text {photons }}=\frac{P_{\text {pixel }} \Delta t_{d w e l l}}{\left(\frac{h c}{\lambda}\right)}$

The number of electrons $N_{\text {electrons }}$ read out from a column of $N_{T D I}$ pixels due to the image of the ground element of side length $L$ is therefore given by Equation (10) where $Q_{E}$ is the quantum efficiency of the CCD at the wavelength $\lambda$.

$$
\begin{aligned}
N_{\text {electrons }}= & N_{T D I}\left(\frac{\pi}{4 h c}\right) T_{\text {atm }} T_{\text {scope }} T_{\text {filter }} Q_{E}\left(D^{2}-D_{o}^{2}\right) \\
& L_{\text {rad }}\left(\frac{L^{3} \operatorname{Cos} \phi}{\mathrm{H}^{2} v_{g}}\right) \lambda \Delta \lambda
\end{aligned}
$$

The signal to noise ratio SNR is given by Equation (11):

$$
S N R=\frac{N_{\text {electrons }}}{\sqrt{N_{\text {electrons }}^{2}+N_{\text {readout }}^{2}+N_{\text {dark }}^{2}}}
$$

$N_{\text {shot }}$ is the shot noise due to the random nature of the receipt of photons and is equal to the square root of $N_{\text {electrons }}$. Readout noise is the noise introduced by the amplifier when the electron signal (current) from a TDI column is read out from the CCD. The dark signal is due to thermally created electrons and is proportional to the number of TDI stages. $N_{\text {dark }}$ is the shot noise in the dark signal and is equal to the square root of the number of electrons in the dark signal ${ }^{(19)}$.

For the CCD5061 the typical readout noise is 40 electrons and the dark signal is typically $46 \cdot 8$ electrons for 8 TDI stages ${ }^{(18)}$. The SNR is therefore given by Equation (12):

$$
S N R=\frac{N_{\text {electrons }}}{\sqrt{N_{\text {electrons }}+1,647}}
$$

It is assumed that a backthinned version of the CCD5061 can be obtained with quantum efficiencies similar to the Hamamatsu S1020208 TDI CCD. These quantum efficiencies are shown in Table 4 and used in the calculation of the SNR. The Hamamatsu S10202-08 backthinned TDI-CCD has high quantum efficiency from UV to NIR with 4,160 pixels of $12 \mu \mathrm{m}$ width and 128 stage TDI $^{(20)}$.

Using Equation (10) with $N_{T D I}=8, T_{\text {scope }}=0.85$ for the Questar 3.5 with MgFl coatings on the refractive elements and silver coated mirrors $^{(15)}, T_{\text {atm }}=0.7, T_{\text {filter }}=0.6, D=89 \mathrm{~mm}$ and $d=27.9 \mathrm{~mm}^{(13)}, L=$ $6.5 \mathrm{~m}, H=600 \mathrm{~km}, \varphi=0$ and $v_{g}=6,907 \mathrm{~ms}^{-1}$ the SNR is calculated in five spectral bands (Table 2) with the results presented in Table 4. The spectral bands are those used by RapidEye and the spectral radiances and SNR requirements in these bands are obtained from the GMES Sentinel-2 Mission Requirements ${ }^{(1)}$. It can be seen that the SNR exceeds requirements in all spectral bands. 
Table 4

SNR for the 6U standard CubeSat imaging payload in five spectral bands with $\boldsymbol{N}_{T D I}=8$

\begin{tabular}{|c|c|c|c|c|c|c|}
\hline Band & $\underset{\mathbf{n m}}{\lambda}$ & $\begin{array}{l}\Delta \lambda \\
\mathbf{n m}\end{array}$ & $\boldsymbol{Q}_{E}$ & $\underset{\mathbf{W m}^{-2} \mathbf{s r}^{-1} \boldsymbol{\mu} \mathbf{I}}{\boldsymbol{L}_{\text {ref }}}$ & $\begin{array}{c}\text { SNR@ @ } L_{r e f} \\
\text { (Requirement) }\end{array}$ & SNR \\
\hline Blue & 475 & 70 & $0 \cdot 80$ & 128 & 154 & 170 \\
\hline Green & 555 & 70 & 0.87 & 128 & $168 \cdot 4$ & 192 \\
\hline Red & 658 & 55 & 0.90 & 108 & $142 \cdot 1$ & 173 \\
\hline Red edge & 710 & 40 & 0.89 & $74 \cdot 6$ & 117 & 124 \\
\hline NIR & 805 & 90 & 0.66 & 103 & $174 \cdot 6$ & 206 \\
\hline
\end{tabular}

\subsection{DATA DOWNLINK (14Mbps)}

The requirements for the data downlink (Table 2) will be met by an $\mathrm{S}$ band data communications subsystem with a data downlink rate of greater than $10 \mathrm{Mbps}$, bit error rate $(\mathrm{BER})=10^{-10}$ and a $3 \cdot 7 \mathrm{~m}$ ground station.

A CubeSat S Band transceiver card is specified similar in transmitter functionality to the Isis $38,400 \mathrm{bps} \mathrm{S}$ Band Transmitter $(2 \mathrm{~W}$ input, $1 \mathrm{~W}$ RF output $)^{(21)}$. This card has the dimensions $90 \mathrm{~mm} \times 96 \mathrm{~mm}$ $\times 40 \mathrm{~mm}$ and a mass of $<125$ grams and frequencies of 2,100$2,500 \mathrm{MHz}$ are supported. A frequency of $2,250 \mathrm{MHz}$ is chosen which has a wavelength of $13 \cdot 3 \mathrm{~cm}$. To support higher data rates a card with the same dimensions but $10 \mathrm{~W}$ input and $2 \mathrm{~W}$ RF output can be developed that supports speeds of $5-10 \mathrm{Mbps}$ with three variable speeds $^{(22)}$. It is assumed that even higher speeds of up to $42 \mathrm{Mbps}$ (but with the same input and output power) can be supported by a version of this card, and that a VHF or UHF receiver can be included on the card with little change in dimensions. It will be shown that at this output power and frequency high data rates of up to $42 \mathrm{Mbps}$ are predicted by the link equation when applied to the $6 \mathrm{U}$ CubeSat and the data downlink rate averaged over a ground station pass is $14 \mathrm{Mbps}$.

A $5 / 8 \lambda$ whip monopole antenna is specified although not shown in Fig. 2. The relatively short wavelength results in an antenna only $8.3 \mathrm{~cm}$ in length so it can be easily packaged in the spacecraft. This allows the system to benefit from the higher gain of a $5 / 8 \lambda$ monopole compared to a quarter wave monopole. The gain of a $5 / 8 \lambda$ monopole is around $3 \mathrm{~dB}$.

The link equation can be written in the form of Equation (13). The space loss $L_{s}$ and total system losses $L_{o}$ are placed in the denominator and defined accordingly.

$$
\frac{E_{b}}{N_{o}}=\frac{\operatorname{EIRP}\left(\frac{G}{T}\right)}{k R L_{s} L_{o}}
$$

$E b / N o$ is the received energy per bit divided by the spectral noise density. EIRP is the equivalent isotropic radiated power and is equal to $G_{T} P_{T}$ where $G_{T}$ is the satellite antenna gain and $P_{T}$ is the RF power transmitted by the satellite antenna. $G / T$ is the figure of merit for the ground station which has the specifications of a DMC ground station. The figure of merit for the $3.7 \mathrm{~m} \mathrm{DMC}$ ground station is $13 \mathrm{~dB} / \mathrm{K}$ at an elevation angle of $10^{\circ(4)} . k$ is Boltzmann's constant and $R$ is the bit rate in bps. The space loss is given by Equation (14) where $r$ is the distance in metres between the satellite and the ground station and $\lambda$ is the wavelength.

$$
L_{s}=\left(\frac{4 \pi r}{\lambda}\right)^{2}
$$

The total system losses amount to $7 \cdot 56 \mathrm{~dB}$ and include an antenna pointing loss of $3 \mathrm{~dB}$. The spacecraft transmitted radiation pattern is relatively broad so the pointing losses refer to the ground station, for which the beam width is only $2 \cdot 5^{\circ}$. Other losses include implementation losses (in the ground station electronics chain) of $2 \mathrm{~dB}$, a spacecraft transmitter to antenna loss of $1 \mathrm{~dB}$, a radome loss of $1 \mathrm{~dB}$ (a high latitude ground station is envisioned to downlink data on

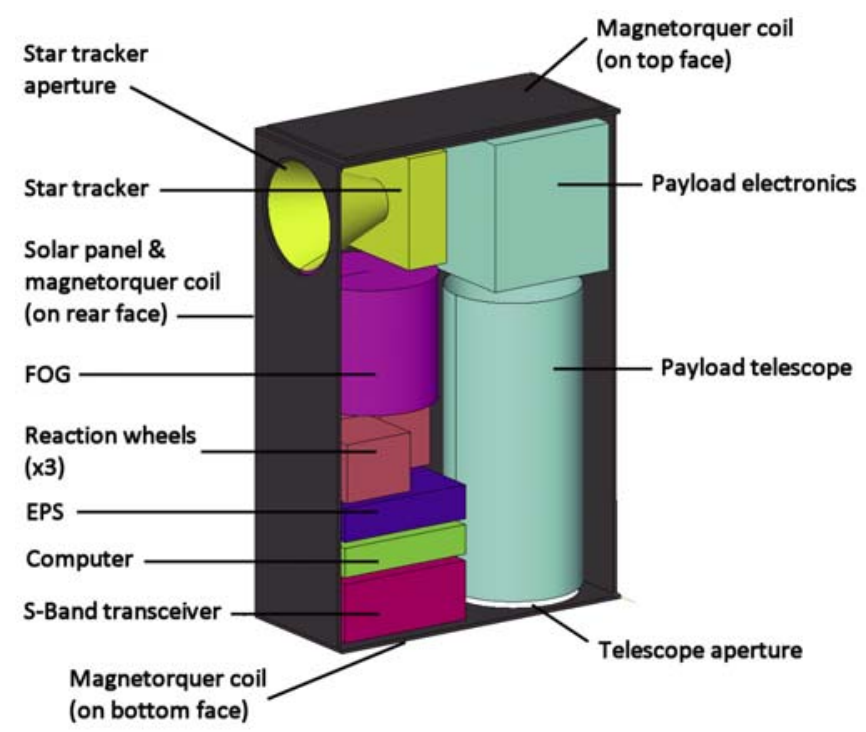

Figure 2. Packaging of the $6 \mathrm{U}$ CubeSat.

each orbit), polarisation losses of $0 \cdot 3 \mathrm{~dB}$ and $0 \cdot 26 \mathrm{~dB}$ atmospheric attenuation. There is no rain fade at $2 \cdot 25 \mathrm{GHz}$.

A value of $12 \cdot 6 \mathrm{~dB}$ for $E b / N o$ would be required for a $\mathrm{BER}=10^{-9}$ if BPSK or QPSK modulation were used without forward error correction $^{(23)}$. To meet the downlink rate requirement a FEC must be used to reduce the Eb/No required. QPSK 8/9 modulation and encoding, part of the DVB-S2 standard, is chosen which reduces the $E b / N o$ required for a $\mathrm{BER}=10^{-10}$ to $4.02 \mathrm{~dB}$, which is a coding gain of $8.6 \mathrm{~dB}$ and within $2.75 \mathrm{~dB}$ of the Shannon limit ${ }^{(24)}$. A $3 \mathrm{~dB}$ link margin is added increasing the required value of $E b / N o$ to $7 \cdot 02 \mathrm{~dB}$.

With FEC the bit rate includes the transmission of error correction bits. The fraction of data bits is $8 / 9$, so the data downlink rate is $8 / 9$ of the bit rate $R$ in Equation (2). The spacecraft is assumed to transmit with three different speeds from elevation angles measured from the ground station of $10^{\circ}-30^{\circ}, 30^{\circ}-60^{\circ}$ and $60^{\circ}-90^{\circ}$ respectively to take advantage of the decreasing space loss as the spacecraft moves closer to the ground station. This increases the data that can be downlinked by almost a factor of 3 compared to using one constant transmit speed determined by the link equation at an elevation angle of $10^{\circ}$. The use of three different speeds at these angles allows the downlink of $69 \%$ of the total data that could be downlinked if a continuously variable transmitter were available.

Using the following values of the variables in Equation (13) the data rate is calculated:

$G_{T}=3 \mathrm{~dB}=2$;

$P_{T}=2 \mathrm{~W}$;

$E I R P=G_{T} P_{T}=4 \mathrm{~W}$

$G / T=13 \mathrm{~dB} / \mathrm{K}=19 \cdot 95 \mathrm{~K}^{-1}$;

$k=1.38 \times 10^{-23} \mathrm{JK}^{-1}$;

$E_{b} / N_{o}=7 \cdot 02 \mathrm{~dB}=5 \cdot 035$

$\lambda=0.133 \mathrm{~m}$

$L_{o}=7 \cdot 56 \mathrm{~dB}=5 \cdot 70$;

$L_{s}\left(10^{\circ}\right)=3.33 \times 10^{16}(165 \mathrm{~dB})$;

$L_{s}\left(30^{\circ}\right)=9.87 \times 10^{15}(160 \mathrm{~dB})$;

$L_{s}\left(60^{\circ}\right)=4.24 \times 10^{15}(156 \mathrm{~dB})$;

$\Rightarrow R_{\text {data }}\left(10^{\circ}\right)=5 \cdot 4 \mathrm{Mbps}, R_{\text {data }}\left(30^{\circ}\right)=18 \cdot 1 \mathrm{Mbps}, R_{\text {data }}\left(60^{\circ}\right)=$ $42 \cdot 2 \mathrm{Mbps}$

The data rate is integrated over a ground station pass to obtain the total data downlinked which is 8.0 Gbits. The spacecraft downlinks data for 9.58 minutes, the time it is visible to the ground station above an elevation angle of $10^{\circ}$. The total data downlinked can be divided by the downlink time to get the average downlink speed of $14 \mathrm{Mbps}$ which satisfies requirements. 
Table 5

Disturbance torques acting on the $6 \mathrm{U}$ CubeSat

$\begin{array}{ll}\text { Disturbance } & \text { Torque } \\ \text { Gravity gradient } & 9.0 \times 10^{-8} \mathrm{Nm} \\ \text { Solar radiation pressure } & 3.1 \times 10^{-8} \mathrm{Nm} \\ \text { Magnetic field } & 4.5 \times 10^{-8} \text { to } 4.5 \times 10^{-6} \mathrm{Nm} \text { for } \\ & D=10^{-3} \text { to } 0 \cdot 1 \mathrm{Am}^{2} \\ \text { Aerodynamic } & 3.4 \times 10^{-8} \mathrm{Nm}\end{array}$

Each spectral band of the imaging payload generates $4,000 \times 12$ bits per dwell time $=48,000$ bits per $0.941 \mathrm{msec}=51 \mathrm{Mbps}$. Five spectral bands therefore generate $255 \mathrm{Mbps}$. Lossless compression of $2: 1$ is available which effectively reduces this to $127.5 \mathrm{Mbps}$. Depending on the overhead in the data downlinked a ground station pass can therefore downlink up to $63 \mathrm{~s}$ worth of imaging data. This corresponds to an imaged area of up to $435 \mathrm{~km} \times 26 \mathrm{~km}=11,310 \mathrm{~km}^{2}$ that can be downlinked per ground station pass. A high latitude ground station would offer a ground station pass each orbit, with only $1 \mathrm{~GB}$ of memory required to store the $8 \mathrm{Gbits}$ of data between imaging and downlink.

\subsection{ATTITUDE CONTROL SYSTEM}

\subsection{Requirements for pointing accuracy and pointing stability}

The pointing accuracy must be sufficient to ensure that a target is within the field of view of the imaging system. It is reasonable to specify an accuracy requirement of one tenth of the FOV to ensure that a targeted area is mostly within the FOV. The FOV is $2.5^{\circ}$ leading to a pointing accuracy requirement of $0.25^{\circ}$. This is also more than sufficient accuracy to point the main solar array towards the Sun when not imaging to maximise power generation.

The use of time delay integration (TDI) by the optical payload to achieve acceptable signal to noise ratios in the different imaging spectral bands compensates for the reduced light gathering capacity of the small aperture. In terms of SNR, TDI allows a small aperture to be functionally identical to a large aperture. However this leads to more demanding requirements for pointing stability.

Use of TDI creates the ACS requirement that an image on a pixel stays within the TDI column as the spacecraft moves down track. It was considered acceptable that the pointing remained within half a pixel width $72 \%$ of the time during simulations for the Mars Reconnaissance Orbiter HiRISE instrument for the stressing case of all three reaction wheels spinning at $3,000 \mathrm{RPM}^{(25)}$. A point source, initially in the middle of the pixel width, would stay within the pixel width $72 \%$ of the time. Therefore approximately 1 sigma $(68.3 \%$ versus $72 \%$ ) pointing stability of half a pixel width was considered acceptable for MRO HiRISE in this stressing case. Three sigma pointing stability of half a pixel width in all cases is used as the requirement for TDI imaging on the $6 \mathrm{U}$ CubeSat. This is equivalent to the requirement that the pointing remain within half a pixel width $99.7 \%$ of the time during TDI for a pixel. Given the example of MRO HiRISE this would seem to be a reasonable requirement.

The angle subtended by the GSD $L$ of $6.5 \mathrm{~m}$ from an altitude $H$ of $600 \mathrm{~km}$ is $10.83 \mu \mathrm{rad}$ or $2.23 \mathrm{arcsec}$. At an altitude of $600 \mathrm{~km}$ the magnitude of the spacecraft velocity projected on the ground is 6907 $\mathrm{ms}^{-1}$ and the dwell time for the pixel corresponding to this GSD is $0.941 \mathrm{~ms}$. Therefore the TDI integration time is $8 \times 0.941 \mathrm{~ms}=$ $7.53 \mathrm{~ms}$. The pointing stability requirement is therefore that a commanded pointing direction change less than 1.18 arcsec in $7 \cdot 53 \mathrm{~ms} 99 \cdot 7 \%$ of the time during imaging.
Table 6

ACS design which satisfies requirements

\section{Sensors}

$1 \times$ Star Tracker

Comtech AeroAstro

Miniature Star Tracker

$<90$ arc second accuracy

in three axes $(1 \sigma)$

Lost in space capability

$6 \mathrm{~cm} \times 7.62 \mathrm{~cm} \times 7.62 \mathrm{~cm}$

$375 \mathrm{~g}$ (not including baffle)

$<2 \mathrm{~W}$

\section{$1 \times$ IMU}

Northrop Grumman LN-200S

Fiber Optic Gyroscope

Angular random walk: $<0 \cdot 07^{\circ} / \sqrt{ }$

Short term bias drift: $<0 \cdot 1 \% \mathrm{hr}$

$400 \mathrm{~Hz}$ data rate

$8.89 D \times 8.64 \mathrm{~cm}$

$748 \mathrm{~g}$

12W@5V

Magnetometers mounted on computer card

\author{
Actuators \\ $3 \times$ orthogonal reaction wheels \\ Sinclair Interplanetary \\ Nanosatellite \\ Reaction Wheels RW-0.03-4 \\ Momentum storage: $30 \mathrm{mNms}$ \\ (a) 5,600 rpm \\ Maximum torque: $2 \mathrm{mNm}$ \\ $5 \mathrm{~cm} \times 5 \mathrm{~cm} \times 4 \mathrm{~cm}$ \\ $185 \mathrm{~g}$ \\ 2.0W@ full torque \\ $0 \cdot 1-0.4 \mathrm{~W}$ steady state \\ $3 \times$ orthogonal magnetorquers: \\ Clyde Space 6U solar panel \\ with embedded wire \\ magnetorquers \\ Embedded wire magnetorquers \\ mounted on orthogonal faces \\ $0.177 \mathrm{Am}^{2}$ for $1 \mathrm{U}$ panel \\ $0.266 \mathrm{Am}^{2}$ for $3 \mathrm{U}$ panel
}

\subsection{Disturbance environment}

The attitude of the $6 \mathrm{U}$ CubeSat is disturbed by solar radiation pressure and the Earth's gravity gradient, magnetic field and residual atmosphere. The torques on the spacecraft due to these effects are summarised in Table 5 . The spacecraft residual dipole moment, $D$, is given as a range of possible values from 0.001 to $0.1 \mathrm{Am}^{2}$.

\subsection{ACS design which meets requirements}

To satisfy the requirements subject to this disturbance environment a suite of sensors and actuators are specified. These are summarised in Table 6

The magnetometers are used to measure the magnetic field for calculating magnetorquer use, and to allow initial attitude acquisition following P-POD deployment. Magnetorquers are specified in the form of wire loops embedded in the main solar panel, and similar panels without the solar cells are mounted on the top and bottom faces of the $6 \mathrm{U}$ CubeSat as shown in Fig. 2, and on the $10 \mathrm{~cm} \times 30 \mathrm{~cm}$ face adjacent to the telescope. The specifications for such panels (with solar cells) are shown in Table 6. The magnetorquers are used to create magnetic dipole moments in orthogonal directions for reaction wheel desaturation.

The orientation of the star tracker is shown in Fig. 2. With this orientation, in a late morning Sun synchronous orbit, the star tracker is not blinded during imaging when the telescope boresight is pointed approximately towards the nadir, nor is there blinding if the telescope boresight is rolled across track. Furthermore, for the remainder of the orbit while the normal to main solar array is kept pointed towards the Sun for maximum power generation the spacecraft can be rotated around the normal to the main solar array to keep the Earth (and Moon) outside of the field of view of the star tracker. 


\subsection{IMU is specified to propagate attitude during TDI period}

The pointing stability requirement cannot be met using the star tracker. The LN-200S is a compact inertial measurement unit with three solid state fibre optic gyroscopes and spaceflight heritage ${ }^{(26)}$. The LN-200S fiber optic gyroscope specified has sufficiently low angular random walk noise and drift bias to allow an approach suggested by Grocott and Carroll ${ }^{(27)}$ where the attitude is propagated throughout the exposure time (in this case the TDI integration period) by integrating the rate output from gyroscope. In this case the star tracker attitude data is not used by the ACS to control the attitude during imaging. The main limitation to using this method is the angular random walk noise in the data from the gyroscope, with the short term bias drift a secondary factor. The 1 sigma pointing error after propagating the output from the gyro for $t$ seconds is given by Equation (15) for the LN-200S, where ARW is the angular random walk noise.

$$
1 \sigma \text { pointing error }=\mathrm{ARW} \sqrt{t}<\frac{4 \cdot 2 \operatorname{arcsec}}{\sqrt{s}} \sqrt{t}
$$

After the pixel TDI integration period of $7.53 \mathrm{~ms}$ the $1 \sigma$ pointing error is 0.36 arcsec. The $3 \sigma$ pointing error (knowledge) is therefore 1.09 arcsec. Pointing accuracy is at best equal to the pointing knowledge, indicating that the requirement that the pointing remain within $1 \cdot 18 \operatorname{arcsec}(3 \sigma)$ during the TDI integration period may be met with suitable control loop design provided microvibrations from the RWA are properly characterised.

\subsection{Pointing stability is affected by RWA microvibrations and control loop rate error}

The pointing stability of the MRO HiRISE instrument, which uses a 128 stage TDI CCD, is affected by high frequency jitter, caused by the RWA, and the low frequency control loop rate error ${ }^{(25)}$. The jitter effect of the RWA is proportional to the square of the wheel speed, and on MRO HiRISE a wheel speed of 3,000rpm produced a jitter of less than $0.5 \mu \mathrm{rad}$ (half a pixel width) $72 \%$ of the time ${ }^{(25)}$. For MRO HiRISE the main ACS challenge was therefore the control loop rate error.

Due to the great differences in spacecraft sizes and the dependence on materials and configuration a direct comparison with MRO HiRISE is impossible. However, the example of MRO HiRISE highlights the areas of concern for the $6 \mathrm{U}$ CubeSat ACS, namely the jitter effect of the RWA and the control loop rate error. Compensation for the disturbance environment (Table 5) during imaging builds up reaction wheel speeds of less than 100rpm on the $6 \mathrm{U}$ CubeSat. The spacecraft can execute a fast roll to point at an offnadir target using the $2 \mathrm{mNm}$ maximum torque capacity of the RWA. The maximum moment of inertia of the 6U CubeSat is approximately $0.087 \mathrm{kgm}^{2}$. Application of the maximum torque for one second builds up a rotational velocity of $1.3 \%$ s for the $6 \mathrm{U}$ CubeSat and results in a reaction wheel speed of 373rpm. However this reaction wheel speed is reduced to zero as the spacecraft rotation is cancelled when the desired off-nadir pointing direction is achieved. Therefore reaction wheel speeds remain typically below 100rpm during imaging.

Even so, microvibrations due to the RWA is an area that will require significant attention to properly characterise the effect on pointing stability on this new platform. Attention should also be paid to the control loop rate error.

\subsection{Band-to-band co-registration by image processing}

A transmission filter rather than a dispersive filter is proposed given the limited volume available. A transmission filter works by only passing light in a certain wavelength band, with multiple filters (one for each imaged band) lined up in the direction along track with a CCD behind each filter. As the spacecraft moves along track the image of a spot on the ground moves past each filter, being imaged in each band successively. For the $6 \mathrm{U}$ CubeSat the time taken in seconds for the image of a spot to move between two filters is approximately equal to the distance between the filters in centimetres. Therefore depending on packaging a time difference on the order of seconds can elapse between the image of a spot being acquired in the wavelength band of the first filter and the last. In the case of RapidEye the time difference is 3 seconds.

The approach used for RapidEye is proposed ${ }^{(1)}$, where ground processing after image acquisition is used to co-register the images acquired in the multiple bands. The images in each band are placed in a digital terrain model and then correlated to achieve sub-pixel coregistration.

Geolocation of the images is assumed through the use of GPS onboard the $6 \mathrm{U}$ CubeSat. The GPS chip is assumed to be mounted on the computer card. Thermo-elastic distortion of the spacecraft bus must be considered since this could lead to an angular displacement between the expected telescope boresight and the actual boresight direction. If this were a significant angle this could affect the geolocation accuracy and even the ability to properly frame a target within the swath. However given the small size of the spacecraft thermoelastic distortion is expected to be small.

\subsection{POWER SUBSYSTEM (17.7 W EOLI $20 \mathrm{WH}$ )}

Imaging is limited to around one minute per orbit, assuming all the data is to be downlinked during the next ground station pass. At $600 \mathrm{~km}$ altitude the orbital period is $96.69 \mathrm{~min}$ and the maximum eclipse time is $35.49 \mathrm{~min}$. Therefore the largest $\sim 30 \mathrm{~cm} \times 20 \mathrm{~cm}$ face of the $6 \mathrm{U}$ CubeSat can be kept pointing towards the Sun for most of the sunlit phase to maximise power generation from the solar array on this face. Clyde Space GaAs solar cells which have the dimensions $39.7 \mathrm{~mm} \times 69.1 \mathrm{~mm}$ with a $5 \mathrm{~mm}$ corner crop and an efficiency of $28 \%{ }^{(28)}$ are specified. With these dimensions 20 cells can be located on the $\sim 30 \mathrm{~cm} \times 20 \mathrm{~cm}$ face in a $4 \times 5$ array (oriented with the long axis of the solar cell along the $\sim 30 \mathrm{~cm}$ spacecraft axis). These cells would be mounted on a board embedded with magnetorquer wire loops with an overall thickness of $3.6 \mathrm{~mm}^{(28)}$. The power generation capacity of this array is $20.4 \mathrm{~W}$ BOL and $17.7 \mathrm{~W}$ at the end of a five year mission.

The ERC-32 computer processor is specified for the $6 \mathrm{U}$ CubeSat. This will give the spacecraft the ability to support a high level of onboard autonomy. This space rated processor offers good processing capacity (20MIPS at $25 \mathrm{MHz} / 5 \mathrm{~V}$ ) with low power consumption $(1 \mathrm{~W})$ and high radiation resistance (300krad). The processor plus the few GB of mass memory required are mounted onto a computer card with the dimensions $95 \mathrm{~mm} \times 90 \mathrm{~mm} \times 21 \mathrm{~mm}$ and a mass of $125 \mathrm{grams}$.

Table 7 gives the power budget for four different operational modes; imaging, eclipse, downlink and battery charging. During imaging the IMU is used to meet the pointing stability requirement described in Section 5.1, resulting in a total power load of $25.5 \mathrm{~W}$. The reaction wheel speeds are low so the minimum of the quoted power consumption (Table 6, $0 \cdot 1 \mathrm{~W}$ ) for a reaction wheel is assumed. When not imaging the pointing stability requirement does not apply and the IMU is turned off to reduce the power load. Fibre optic gyroscopes have no warm up time ${ }^{(29)}$ so the imaging time is used for the length of time the IMU is powered on. When not imaging the ACS must maintain Sun pointing as described above, and keep the spacecraft antenna direction of maximum gain pointed towards the ground station during the downlink pass. The ACS system should be capable of maintaining pointing with $0.25^{\circ}$ pointing accuracy with the star tracker without data from the IMU and this accuracy is sufficient for Sun and ground station pointing. 
Table 7

Power budget of the $6 \mathrm{U}$ CubeSat

\section{Component/Mode}

Star Tracker

IMU

RWA

Processor

Payload

Heater

Transmitter

Receiver

Solar cells

Total power consumption (generation)

Imaging $(\mathbf{W})$
2
12
$0 \cdot 3$
1
10
0
0
$0 \cdot 2$
0
$25 \cdot 5$

A simple power model can be used to show that power requirements during eclipse, imaging and downlink can be met. During imaging and downlink the main solar array is not kept pointing towards the Sun, since in general different attitudes will be required to point the telescope to the target and the antenna maximum gain direction to the ground station. The worst case is that the main array generates no power during imaging and downlink, requiring the spacecraft batteries to power the spacecraft during these phases as well as during eclipse by the Earth. The power requirement during eclipse is $8.3 \mathrm{~W}$ (Table 7), including $4.8 \mathrm{~W}$ for electrical heating. The eclipse duration is $35.49 \mathrm{~min}$ so the total energy that must be delivered to the spacecraft during eclipse is $4.91 \mathrm{Wh}$. The power requirement during downlink is $13.5 \mathrm{~W}$ (Table 7). The downlink duration is $9.58 \mathrm{~min}$ so the total energy that must be delivered to the spacecraft during downlink is $2 \cdot 16 \mathrm{Wh}$.

RALCam 3 is a $17 \mathrm{~W} 4$ band 10 metre GSD imager with four 8,800 pixel linear CCD detectors of $7 \mu \mathrm{m}$ pixel width ${ }^{(30)}$. With $56 \%$ of the illuminated pixels of Ralcam 3, the $6 \mathrm{U}$ CubeSat imager has a projected power consumption of $10 \mathrm{~W}$. This does not include the power required by the data recorder. However only $1 \mathrm{~GB}$ of data storage is required, which is assumed to be provided by low power solid state memory mounted on the computer card.

The power requirement during imaging is $25.5 \mathrm{~W}$ (Table 7). The downlink duration is $63 \mathrm{~s}$ so the total energy that must be delivered to the spacecraft during downlink is $0.45 \mathrm{Wh}$. The total energy that must be delivered by the spacecraft batteries each orbit is therefore $7 \cdot 52 \mathrm{Wh}$. Assuming the batteries deliver power with $90 \%$ efficiency, the total electrical energy drained from the batteries each orbit is $8 \cdot 36 \mathrm{Wh}$.

This energy must be replaced by charging from the solar array during the sunlit phase of the orbit, when the spacecraft is not imaging or downlinking. The downlink is assumed to occur during the sunlit phase in the worst case leaving $50.57 \mathrm{~min}$ for charging the batteries. In this battery charging mode, the spacecraft must supply $3.3 \mathrm{~W}$ to the ACS system and $0.2 \mathrm{~W}$ to the receiver, leaving $14.2 \mathrm{~W}$ available for charging the batteries for $50.57 \mathrm{~min}$ (11.97Wh of energy into the batteries). Assuming $70 \%$ charging efficiency, the batteries can gain $8 \cdot 38 \mathrm{Wh}$ of electrical energy, fully charging them.

To avoid a high depth of discharge and provide operational flexibility a $20 \mathrm{Wh}$ battery integrated into a Clyde Space CubeSat power system is specified. This power system has the dimensions $95 \mathrm{~mm} \times$ $90 \mathrm{~mm} \times 25 \mathrm{~mm}$ with a weight of $204 \mathrm{~g}$.

It has been assumed that downlink occurs during the sunlit phase, and that no power generation occurs during downlink. A more realistic model will include some power generation during downlink. Assuming the normal to the solar array points on average $60^{\circ}$ away from the direction to the Sun during downlink, $1.4 \mathrm{Wh}$ of energy will be generated by the array during downlink which can be supplied to the batteries. This is equivalent to $0.55 \mathrm{~W}$ of continuous power drawn from the batteries throughout the orbit, providing a margin to account for unbudgeted power items such as the computer card aside from the processor.

Eclipse (W)
2
0
$0 \cdot 3$
1
0
$4 \cdot 8$
0
$0 \cdot 2$
0
$8 \cdot 3$

Downlink (W)
2
0
$0 \cdot 3$
1
0
0
10
$0 \cdot 2$
0
$13 \cdot 5$

Battery charging $(\mathbf{W})$
2
0
$0 \cdot 3$
1
0
0
0
$0 \cdot 2$
$(17 \cdot 7 \mathrm{EOL})$
$(14 \cdot 2)$

\subsection{THERMAL (258 K COLD CASE, 312K HOT CASE)}

As the spacecraft size decreases the ratio of the surface area to the volume increases so it is expected to be more difficult to maintain acceptable temperatures during eclipse. However smaller CubeSats have already successfully flown in space indicating thermal management, particularly in the cold case, of such small spacecraft is possible. A variable emissivity film is considered for the $6 \mathrm{U}$ CubeSat to optimise thermal management. A simple thermal model of the spacecraft is made by assuming the spacecraft is isothermal and in thermal equilibrium with its surroundings. The thermal emission from the spacecraft is balanced by the radiative energy absorbed by the spacecraft (direct sunlight, Earth scattered visible light and thermal radiation from the Earth) and the electrical power dissipated in the spacecraft as heat. The spacecraft is assumed to have one $\sim 30 \mathrm{~cm} \times$ $20 \mathrm{~cm}$ face covered with solar cells with an emissivity of 0.8 and absorptivity of 0.7 . The other faces are assumed to be covered with a film of variable emissivity which allows an emissivity of 0.15 to 0.68 $(0.53$ variation in emittance) to be selected by application of a small amount $(<64 \mathrm{~mW})$ of electrical power ${ }^{(31)}$. While this film has not yet flown in space it has completed nearly the entire range of space qualification tests ${ }^{(32)}$. The film is assumed to have an absorptivity of 0.2 . During eclipse the film is set to an emissivity of $0 \cdot 15$ to minimise heat loss from the spacecraft, and in the sunlit phase the film is set to an emissivity of 0.68 to minimise the temperature of the spacecraft.

As mentioned in the previous section $4.8 \mathrm{~W}$ of heating is assumed during eclipse. The solar array is pointed towards the Earth during eclipse to maximise the absorption of heat emitted by the Earth as infrared radiation. The minimum temperature during eclipse (the cold case $)$ is $258 \mathrm{~K}\left(-15^{\circ} \mathrm{C}\right)$.

During the sunlit phase thermal equilibrium will not be reached during the $63 \mathrm{~s}$ imaging mode. Thermal equilibrium is possible during the battery charging mode, and possibly the downlink mode. In the battery charging mode, the total amount of heat generated onboard the $6 \mathrm{U}$ CubeSat is $7.76 \mathrm{~W}$ including the heat generated from recharging the batteries. In downlink mode the total amount of heat generated onboard is $11.5 \mathrm{~W}$ including $8 \mathrm{~W}$ dissipated in the transmitter. Assuming thermal equilibrium in both cases the maximum temperature in each mode (the hot case) is $308 \mathrm{~K}\left(35^{\circ} \mathrm{C}\right)$ and $312 \mathrm{~K}\left(39^{\circ} \mathrm{C}\right)$.

The results of this simple isothermal model suggest that by placing the heater near the batteries acceptable thermal management of the spacecraft can be achieved.

\subsection{DIMENSIONS, STRUCTURE AND PACKAGING}

The $6 \mathrm{U}$ CubeSat has a $2.5 \mathrm{~mm}$ aluminium skin and its internal components are packaged as shown in the cutaway drawing in Fig. 2. The mass budget is given in Table 8 showing that the total mass of 
Table 8

Mass budget of the $6 \mathrm{U}$ CubeSat

$\begin{array}{ll}\text { Component } & \text { Mass (kg) } \\ \text { Star tracker } & 0 \cdot 375 \\ \text { IMU } & 0 \cdot 748 \\ \text { RWA } & 0 \cdot 555 \\ \text { EPS and batteries } & 0 \cdot 204 \\ \text { Computer } & 0 \cdot 125 \\ \text { Transceiver } & 0 \cdot 080 \\ \text { Structure (includes 20\% margin for fasteners } & 1 \cdot 920 \\ \text { and launch rails) } & \\ \text { Solar panels (including embedded magnetorquers) } & 0 \cdot 297 \\ \text { Deorbit device } & 0 \cdot 080 \\ \text { System total } & 4 \cdot 38 \\ \text { Payload - telescope } & 1 \cdot 4 \\ \text { Payload - electronics } & 1 \cdot 0 \\ \text { Margin } & 1 \cdot 22 \\ \text { Total } & 8 \cdot 00\end{array}$

the spacecraft without the payload is $4.38 \mathrm{~kg}$, and $6 \cdot 78 \mathrm{~kg}$ including the payload. The payload electronics are bespoke and are assumed to have approximately the density of water, leaving a margin of $1.22 \mathrm{~kg}$. The CubeSat standard specifies the dimensions of a $3 \mathrm{U}$ CubeSat to be $100 \mathrm{~mm} \times 100 \mathrm{~mm} \times 340 \cdot 5 \mathrm{~mm}$ with a maximum mass of $4 \mathrm{~kg}$, where $340.5 \mathrm{~mm}$ is the length of the rails that interface with the PPOD launcher and the actual body length is $327.5 \mathrm{~mm}^{(33)}$.

No standard is defined for a $6 \mathrm{U}$ CubeSat. It has been suggested that the $6 \mathrm{U}$ CubeSat is specified so that a loaded 6U P-POD launcher has the same dimensions and mass properties as two loaded $3 \mathrm{U}$ PPOD launchers mounted side by side ${ }^{(3)}$. This implies $6 \mathrm{U}$ CubeSat dimensions of $100 \mathrm{~mm} \times \sim 220 \mathrm{~mm} \times 340.5 \mathrm{~mm}$ with an actual body length of $327.5 \mathrm{~mm}$ and a maximum mass of $8 \mathrm{~kg}$, where the width is slightly wider than $200 \mathrm{~mm}$ because of the gap between the vertices of the adjacent launch rails of two side by side P-PODs. While this is a reasonable suggestion, to be conservative the $6 \mathrm{U}$ CubeSat presented in this paper has the dimensions of two $3 \mathrm{U}$ CubeSats side by side, namely $100 \mathrm{~mm} \times 200 \mathrm{~mm} \times 340.5 \mathrm{~mm}$ with a body length of $327.5 \mathrm{~mm}$ and a mass less than or equal to $8 \mathrm{~kg}$. This is presumably the lower limit of the dimensions which will eventually be specified for the $6 \mathrm{U}$ CubeSat.

\subsection{CONCLUSIONS}

A system design has been described which will enable a $6 \mathrm{U}$ CubeSat to carry out a commercial Earth observation mission competitive with the DMC and RapidEye spacecraft. The following conclusions are drawn:

A bespoke optical design is recommended, although the performance of a COTS telescope suggests requirements for GSD, swath and MTF can be met.

A fiber optic gyroscope can be used to propagate the attitude during TDI imaging to achieve the necessary pointing stability

Coding gain and a three speed transmitter enable the spacecraft to achieve an average $14 \mathrm{Mbps}$ downlink rate at S-Band to a $3.7 \mathrm{~m}$ ground station.

Further work is suggested to characterise the effect of microvibrations from the reaction wheel assembly on the pointing stability and to model the ACS control system to quantify the control loop rate error.

\section{REFERENCES}

1. Nason, I., Creedon, M. and Johansen, N. CUBESAT P-Pod Deployer Requirements, 2002. <http://cubesat.atl.calpoly.edu/media/Documents/ Launch\%20Providers/ppod_mk1_icd.pdf $>$
2. ESA, Cubesats. <http://www.esa.int/esaMI/Education/SEM3N03MD AF $0 . h t m l>$

3. CROоK, M. NPS CubeSat Launcher Design, Process and Requirements, 2009. <http://www.dtic.mil/cgi-bin/GetTRDoc?AD=ADA501503\& Location $=\mathrm{U} 2 \& \mathrm{doc}=\mathrm{GetTRDoc} . \mathrm{pdf}>$

4. Curiel, A., Boland, L., Cooksley, J., Berhti, M., Stephens, P., Sun, W. and SweEting, M. First results from the disaster monitoring constellation (DMC), Acta Astronautica, 2005, 56, pp 261-271.

5. Teston, F., Bernaerts, D. and Gantois, K. Proba, an ESA technology demonstration mission, results after 3 years in orbit, 2004, Proceedings of the 4S Symposium: Small Satellites, Systems and Services, ESA SP571, 2004, pp 7.1-7.10.

6. WertZ, J.R. Spacecraft Attitude Determination and Control, 1978, D. Reidel Publishing Company, Holland.

7. Johnson, N.L. and StansBery, E.G. The new NASA orbital debris mitigation procedural requirements and standards, Acta Astronautica, 2009, 10.1016/j.actaastro.2009.07.009.

8. Tethers Unlimited, End-of-mission deorbit module for nanosatellites. $<$ http://www.tethers.com/SpecSheets/nanoTerminator.pdf $>$

9. Surrey Satellite Technology Ltd, Wide swath DMC MSI datasheet. $<$ http://www.sstl.co.uk/assets/Downloads/DMC\%20MSI.pdf $>$

10. MDA, RapidEye - The first six months in orbit, IAA-B7-1501, 2009. $<$ http://media.dlr.de:8080/erez4/erez? $\mathrm{cmd}=$ get\&src $=$ os/IAA/archiv7 $/ \mathrm{Pr}$ esentations/1501_MDA\%20RapidEye $\% 20$ Pres entation $\% 20 \mathrm{May} \% 202009 \% 20 \mathrm{IAA} \% 20$ Conference $\% 20$ Berlin.pdf>

11. RapidEye Standard Image Product Specifications, $<$ http://www.rapideye.de/upload/documents/PDF/RE_Product_Specificatio ns_ENG.pdf $>$

12. ESA Sentinel-2 Team, GMES Sentinel-2 Mission Requirements Document, EOP-SM/1163/MR-dr, 2007. <http://esamultimedia. esa.int/docs/GMES/GMES_Sentinel2_MRD_issue_2.0_update.pdf>

13. Questar Corporation, Questar Field Model Telescope Specification Sheet. $<$ http://www.questarcorporation.com/QuestarPDF/FieldModel.pdf $>$

14. Company 7, Questar 50th Anniversary 3-1/2 Telescope. $<$ http://www.company7.com/questar/telescopes/que50thanniversary.html $>$

15. Questar Corporation, 2009, personal communication.

16. PERKINS, J. Questar Corporation, 2009, personal communication.

17. Verdone, P. A Telescope Suitable for Rocket Borne Instrumentation, 1966. <http://ntrs.nasa.gov/archive/nasa/casi.ntrs.nasa.gov/1967000 9591_1967009591.pdf>

18. Fairchild Imaging, 2009. <http://www.fairchildimaging.com/ documents/CCD5061DataSheetRevB_004.pdf >

19. Allan, G. and Flood, C. High-speed, high-resolution, color CCD image sensor, DALSA Inc. <http://www.machinevisiononline.org/ public/articles/dalsa5.pdf $>$

20. Hamamatsu, TDI-CCD image sensors, 2009. <http://sales. hamamatsu.com/assets/pdf/parts_S/s10200-02_etc_kmpd1098e04.pdf>

21. Clyde Space, 2009. <http://www.clyde-space.com/products/ 178 transmitter?page $=3>$

22. TATE, C. MESL, 2009, personal communication.

23. Maral, G. and Bousquet, M. Satellite Communications Systems, 2002, Wiley, UK.

24. The Consultative Committee for Space Data Systems, DVB-S2 Coding \& Modulation Standard Use For High Data Rate Tm Links, 2007, pp 4-3. $<$ http://cwe.ccsds.org/sls/docs/SLS-CandS/Meeting\%20Materials/2007/Spring +Summer_Material/DVB-S2_orange book_withoutmark (July2007).pdfs

25. Lee, S.W. and Skulsky, E.D. Mars Reconnaissance Orbiter design approach for high-resolution surface imaging, 2003, 26th Annual AAS Guidance and Control Breckenridge, CO, USA.

26. LN-200S Inertial Measurement Unit <ftp://ftp.uni-duisburg.de/ Hardware/Litton/Prodsheet_LN200S.pdf>

27. Grocott, S.C.O. and CARROLL, K.A. Arc-Second Attitude Control for the NESS Asteroid/Satellite Tracking Microsat, <http://citeseerx.ist. psu.edu/viewdoc/download?doi=10.1.1.133.7848\&rep=rep1\&type=pdf $>$

28. Greenland, S. Clyde Space, 2009, personal communication.

29. Gupta, B.D. Fiber Optic Sensors: Principles and Applications, 2006, New India Publishing Agency, New Delhi.

30. MDA Orbital Optics, Ralcam 3, <http://www.orbitaloptics.co.uk /products/ralcam-3.php >

31. Ashwin Ushas Corporation, Variable Emittance Electrochromic Material for Spacecraft Thermal Control. <http://www.ashwinushas.com/EleHome/SpaceThermal/SpaceThermal/spacethermal.html>

32. Chandrasekhar, P. Ashwin Ushas Corporation, 2009, personal communication.

33. CubeSat Design Specification Rev. 12, The CubeSat Program, Cal Poly SLO. <http://cubesat.atl.calpoly.edu/media/CDS_rev12.pdf > 